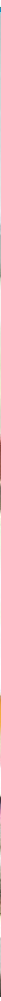

\title{
Supportivtherapie - unverzichtbar in der Onkologie
}

\author{
Interview mit Prof. Dr. Karin Jordan, Klinik für Hämatologie, \\ Onkologie, Rheumatologie am Universitätsklinikum Heidelberg
}

\section{S3-Leitlinie Supportivtherapie}

Die vollständige S3-Leitlinie Supportive Therapie bei onkologischen PatientInnen finden Sie online: https://www.awmf.org/uploads/ tx_szleitlinien/032-0540LI_S3_Supportiv_2017-05.pdf

Als leitende Oberärztin am Universitätsklinikum Heidelberg hat sich Prof. Dr. med. Karin Jordan der Supportivtherapie von Krebspatienten verschrieben. Eines Ihrer Ziele ist es, ein individualisiertes und praktikables Begleitkonzept für Nebenwirkungen moderner Tumortherapie zu entwickeln und allseits zugänglich zu machen.

best practice onkologie: Kaum eine Arbeitsgemeinschaft, die sich mit Supportivtherapie befasst, kommt ohne Sie aus: So sind Sie u. a. stellvertretende Sprecherin der
AGSMO der DKG. Wie bewältigen Sie diesen Arbeitsaufwand?

K. Jordan: Organisation ist alles. Als leitende Oberärztin in Heidelberg muss ich zwar ein breites logistisches Spektrum abdecken, trotzdem bleibt die klinische Arbeit direkt im Patientenkontakt mein Antrieb. Hier sind der Nutzen und die Notwendigkeit unterstützender Maßnahmen direkt spürbar. Die Supportivtherapie begleitet mich schon über Jahre, als Forschungsschwerpunkt in der Klinik, aber auch auf nationaler und internationaler Ebene.

Gelungene Supportivtherapie kommt ohne ein interdisziplinäres Team, das multiple Professionen vereint, nicht aus. Und auch Änderungen in der nationalen Versorgung kann man nur als Team schaffen - hier möchte ich kurz auf Dr. Franziska Jahn hinweisen, meine ehemalige Kollegin am Universitätsklinikum Halle, mit der ich u. a. die S3-Leitlinie Supportiv- therapie [1] koordinierte. Eine elementare Wegbereiterin der Supportivtherapie ist Frau Prof. Dr. Petra Feyer, die seit vielen Jahren als Sprecherin der AGSMO fungiert und als Grande Dame der Supportivtherapie gilt. Natürlich habe ich auch in Heidelberg in den letzten 2 Jahren ein sehr gutes Team aufgebaut. Gemeinsam etablieren und entwickeln wir hier Supportivtherapie weiter.

best practice onkologie: Seit 2017 sind Sie Sektions-Editor für die ESMO-Leitlinien $z u$ „Supportive and Palliative care". Sie fordern im ESMO-Positionspapier zur Supportiv- und Palliativtherapie [2] einen Ansatz der "patient-centred care“. Was verstehen Sie darunter?

K. Jordan: Der behandelnde Onkologe ist natürlich zu Recht primär darauf fokussiert, die bestwirksamste Antitumortherapie zu geben, mit dem Ziel der Heilung bzw. der Lebensverlängerung. Neben der 
Tumorbehandlung ist es aber auch notwendig, Nebenwirkungen vorzubeugen, auftretende Symptome zu behandeln und Hilfe in der Auseinandersetzung mit der Krebserkrankung per se anzubieten. Patienten brauchen - zu verschiedenen Zeitpunkten, in individuellem Maße - z. B. psychoonkologische, physiotherapeutische, soziale, existenzielle oder spirituelle Unterstützung und Begleitung, die auch die Rehabilitation einschließt. „Patient-centred care" bedeutet, dass der $\mathrm{Pa}$ tient mit seinen individuellen Bedürfnissen im Mittelpunkt der Behandlung steht. Der behandelnde Arzt allein kann nicht alle „Baustellen“ bedienen. Hierfür ist ein multidisziplinäres Team aus Ärzten, Pflegenden und Therapeuten jeder Fachrichtung nötig, um dem Patienten gerecht zu werden. „Patient-centred“ heißt aber auch, die Betroffenen selbst aktiv in die Entscheidungsfindungen einzubinden.

\section{》) Beim Ansatz „patient- centred care" wird der Patient in den Mittelpunkt gesetzt}

best practice onkologie: Können Sie für den Bereich der klassischen supportiven Therapie ein praktisches Beispiel geben?

K. Jordan: Die Schlüsselinterventionen beim klassischen Nebenwirkungsmanagement sind das Assessment, die Prophylaxe und die Behandlung von Symptomen. Schon im Vorfeld sollten regelmäßig körperliches Befinden und mögliche Nebenwirkungen abgefragt werden, um entsprechend reagieren zu können. Dazu kann es sinnvoll sein, Symptome über etablierte Fragebögen im Sinne von „patient reported outcomes" zu monitorieren - der Betroffene ist es ja, der z. B. eine veränderte Sensibilität im Mundraum wahrnimmt, deutlich bevor Rötungen einer Mukositis klinisch sichtbar werden. Entscheidend ist dann, konsequent $\mathrm{zu}$ reagieren und die auftretenden Symptome zu behandeln.

best practice onkologie: Die Prophylaxe und Therapie von Übelkeit und Erbrechen unter medikamentöser Tumortherapie sind Ihr Steckenpferd. Bereits in Ihrer Habilitation haben Sie sich damit beschäftigt. Gibt es mittlerweile effektivere Ansätze?
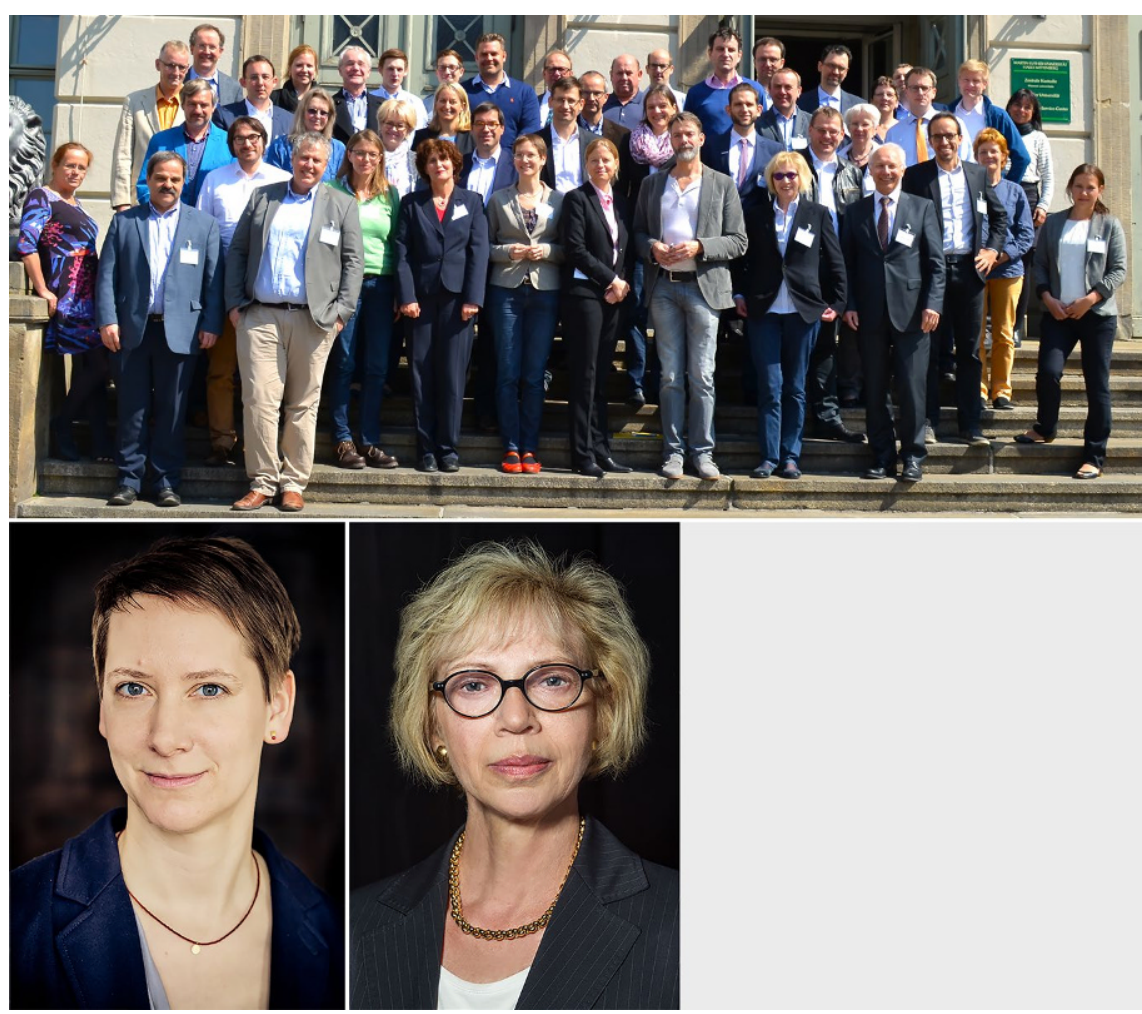

Im Team lässt sich mehr erreichen, wie Prof. Karin Jordan betont. So koordinierte sie gemeinsam mit PD Dr. med. Franziska Jahn (links), Universitätsklinikum Halle, die S3-Leitlinie Supportivtherapie. Eine elementare Wegbereiterin der Supportivtherapie ist außerdem Prof. Dr. Petra Feyer (rechts), Sprecherin der AGSMO

K. Jordan: Nach wie vor unverändert ist die perfekte Prophylaxe wesentlich, um das Auftreten von Übelkeit und Erbrechen möglichst zu verhindern. Dazu sollten all die Antiemetika, über die wir bereits verfügen, auch wirklich eingesetzt werden - natürlich in der an das emetogene Potenzial angepassten Kombination. Mit Olanzapin hat sich hier in den letzten Jahren eine weitere Prophylaxeoption erschlossen. Das atypische Neuroleptikum wird als Rescue-Antiemetikum eingesetzt, kann aber auch prophylaktisch bei Chemotherapien verabreicht werden, deren emetogenes Potenzial als hoch einzustufen ist. Das heißt, dass Patienten mit hoch emetogener Chemotherapie dann neben der Dreifachkombination aus $\mathrm{NK}_{1}-\mathrm{Re}$ zeptor-Antagonist, 5- $\mathrm{HT}_{3}$-Rezeptor-Antagonist und Dexamethason zusätzlich Olanzapin erhalten. Das sind 4 Medikamente zur besseren Kontrolle eines Symptoms. Dazu kommen evtl. noch Schmerztherapie, Hausmedikation und die Tumortherapie selbst. Hier ist sicher kritisches Hinterfragen angesagt. Denn zur optimalen Patientenversorgung gehört auch der Blick auf mögliche Medikamen- teninteraktionen und mögliche Nebenwirkungen durch die Supportivtherapie.

best practice onkologie: Mit leitliniengerechter Behandlung lassen sich Übelkeit und Erbrechen bei mehr als $80 \%$ der $\mathrm{Pa}$ tienten unter hoch emetogener Tumortherapie verhindern [3]. Was ist mit den restlichen $20 \%$ ?

K. Jordan: Das ist eine gute Frage - wenn man sich aber verdeutlicht, in wie vielen unterschiedlichen Situationen oder Symptomkonstellationen Übelkeit als Begleitphänomen auftritt, nicht verwunderlich. „Übel vor Aufregung“, „übel vor Schmerz“, aber auch Übelkeit bei Schleimhautveränderungen wie der einer Gastroenteritis oder Mukositis seien hier nur als Beispiele genannt. Vielfältigste Mechanismen sind bei der Entstehung dieses Phänomens beteiligt.

Wenn Übelkeit und Erbrechen trotz antiemetischer Prophylaxe unzureichend kontrolliert sind, können Rescue-Medikamente wie z. B. Neuroleptika und Dopamin-Rezeptor-Antagonisten möglicherweise Linderung verschaffen. Bei ängstlichen Patienten können Benzodi- 
azepine hilfreich sein. Auch die psychosozialen Faktoren lassen sich optimieren, so entspannt eine räumlich ansprechende Umgebung manchen Patienten. Aber letztlich muss man sagen, dass hier noch weiterer Forschungsbedarf besteht und chanismen, insbesondere gegen Übelkeit, entwickelt werden müssen. Ein interessanter Ansatz wurde auf der MASCC-Jahrestagung 2019 vorgestellt. Dort konnte durch Inhibition der Glykogensynthasekinase 3 - zumindest bei Spitzmäusen ein neuer Angriffspunkt für künftige Antiemetika identifiziert werden [4].

best practice onkologie: Warum sprechen manche Patienten nicht auf die Antiemese an?

K. Jordan: Das ist noch unzureichend verstanden. Sehr wahrscheinlich ist dies im Pathomechanismus begründet und impliziert, dass es weitere Neurotransmitter gibt, die wir noch nicht kennen. Nicht selten haben Patienten tatsächlich keine opneue Antiemetika mit neuen Wirkme-

timale antiemetische Prophylaxe bekommen. Wir müssen unbedingt noch stärker die individuellen Risikofaktoren bedenken, wie junges Alter, Ängstlichkeit oder psychosoziale Faktoren, in Analogie zum „Total Pain“-Konzept von Cicely Saunders. Wenn wir in allen Bereichen verschiedene Erfahrungsebenen von Patienten - körperlich, seelisch, sozial und spirituell - berücksichtigen, dann kann man durchaus eine noch bessere Prophylaxe erreichen.

\section{I) Es müssen weitere Anti- emetika mit neuen Wirk- mechanismen entwickelt werden, die besonders bei Übelkeit wirksam sind}

best practice onkologie: 2017, 2015 und 2010 waren Sie Boardmember der „Consensus Conference on Antiemetic Therapy“

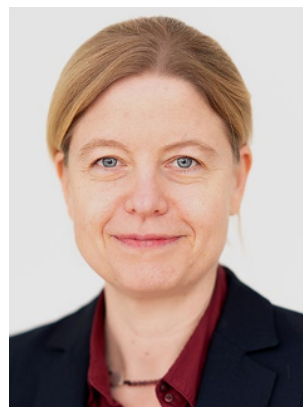

\section{Zur Person:}

Prof. Dr. med. Karin Jordan absolvierte ihr Medizinstudium an der Martin-Luther-Universität in Halle-Wittenberg. Dort schloss sie ihre Facharztausbildung für Innere Medizin, Hämatologie und Onkologie ab und erlangte die Zusatzbezeichnung Palliativmedizin. 2008 wurde sie "associate chair" der Arbeitsgemeinschaft (AG) Supportive Therapie in der AG internistische Onkologie (AIO) der DKG (Deutsche Krebsgesellschaft). 2009 habilitierte sie sich zum Thema Chemotherapieinduziertes Erbrechen in Halle (Saale) und wurde zur Privatdozentin ernannt. Die Supportivtherapie bei Krebspatienten ist bis heute ihr Spezialgebiet. 2010 wurde sie Oberärztin und 2 Jahre später leitende Oberärztin an der Klinik für Innere Medizin IV, Hämatologie und Onkologie am Universitätsklinikum Halle, zudem wurde sie 2014 zur außerplanmäßigen Professorin berufen. 2017 wechselte sie als leitende Oberärztin an die Klinik für Hämatologie, Onkologie, Rheumatologie des Universitätsklinikums Heidelberg, wo sie nun auch die Arbeitsgruppe der AG Supportive Therapien leitet. Seit 9 Jahren verstärkt sie den Vorstand der AG Supportive Maßnahmen in der Onkologie, Rehabilitation und Sozialmedizin (ASORS, jetzt AGSMO) der DKG. Sie ist Sprecherin der AG Supportive Therapie der AG Internistische Onkologie (AIO) in der DKG und Sprecherin der ESMO (European Society of Medical Oncologists) für den Bereich „supportive and palliative care“ sowie die Leitlinienverantwortliche der ESMO für den Bereich Supportivtherapie. In 2020 wurde sie zum Educational Chair der ESMO ernannt. Prof. Jordan koordinierte die erste S3-Leitlinie Supportive Therapie bei onkologischen PatientInnen im Leitlinienprogramm Onkologie. Regelmäßig war sie in den vergangenen Jahren Boardmember der Consensus Conference on Antiemetic Therapy von MASCC (Multinational Association of Supportive Care in Cancer)/ESMO (European Society of Medical Oncology) und ASCO (American Society of Clinical Oncology). E-Mail: karin.jordan@med.uni-heidelberg.de von MASCC/ESMO und auch der ASCO. Gleichen sich die Inhalte der internationalen Leitlinien oder gibt es entscheidende Abweichungen?

K. Jordan: Es gibt in der Tat in den Leitlinien kleinere Abweichungen. Die Leitliniengremien bestehen ja immer aus vielen verschiedenen Personen, und da unterscheiden sich die Meinungen und damit auch der Entscheidungsprozess in kleinen Feinheiten, auch wenn die gleiche Evidenz bewertet wird. Aber die Grundzüge der Leitlinien sind im Wesentlichen gleich.

\section{》) Häufig ist es so, dass Patienten keine optimale antiemetische Prophylaxe bekommen}

best practice onkologie: Welche Nebenwirkungen belasten den Patienten unter Immuntherapie am meisten und wie lassen sich die Toxizitäten managen?

K. Jordan: Das Nebenwirkungsspektrum bei den Immuntherapien unterscheidet sich deutlich von dem der konventionellen Chemotherapien. Hier stehen autoimmune Nebenwirkungen im Vordergrund, häufig sind hier Kolitiden, Diarrhö, Endokrinopathien, Hauttoxizitäten, aber auch neurologische Nebenwirkungen $\mathrm{zu}$ beobachten. Wichtig zu wissen ist, dass die Komplikationen mehrheitlich nicht unmittelbar nach der Immuntherapie auftreten, sondern meist in einem Abstand von mehreren Wochen. Das kann für die Patienten sehr belastend sein und ist eine Herausforderung für den Behandler. Bedeutsam ist, diese Nebenwirkungen zu erkennen, damit sie effektiv behandelt werden können. Dann kann die Immuntherapie oft zeitnah fortgesetzt werden, und ein frühzeitiger Therapieabbruch wird verhindert. Das Nebenwirkungsmanagement bei Immuntherapien soll bei einer Neuauflage der S3-Leitlinie Supportivtherapie Bestandteil sein.

best practice onkologie: Warum ist der S3-Leitlinien-Prozess in den meisten Fällen so langwierig?

K. Jordan: Das ist dem besonderen Anspruch an die S3-Leitlinien geschuldet, sowohl evidenzbasiert als auch durch ein 


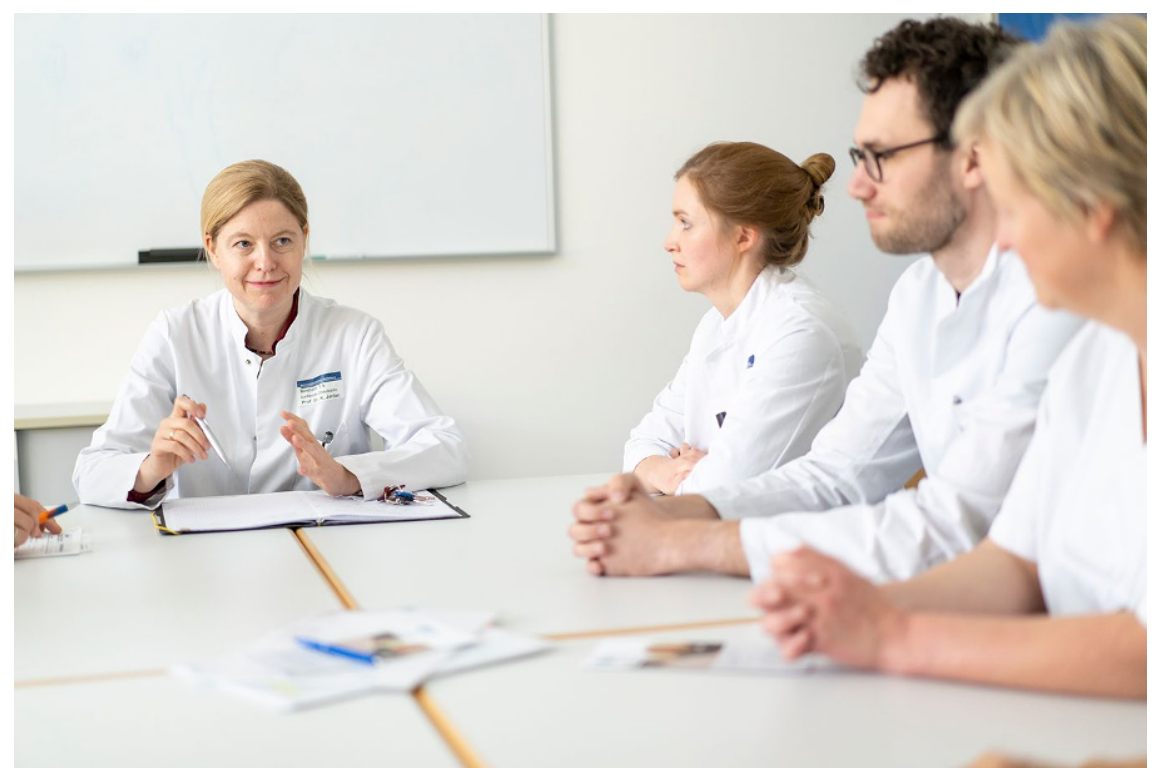

Supportivtherapie erfordert die Arbeit im Team

repräsentatives Gremium erarbeitet worden zu sein. An der Entstehung der S3Leitlinie Supportivtherapie waren insgesamt 70 Mandatsträger und 30 Experten beteiligt. Die gesamte Literatur zu den einzelnen Themen musste systematisch recherchiert und evidenzbasiert aufgearbeitet werden. Die extrahierte Literatur wurde an die einzelnen Arbeitsgruppen gegeben, dort wurden die Empfehlungen erarbeitet und abschließend in Konsensuskonferenzen mit allen Mandatsträgern diskutiert und konsentiert. Das Leitlinienprogramm Onkologie arbeitet daran, Leitlinien besser an die Anforderungen der Informationsgesellschaft anzupassen, auch um diese Prozesse zu beschleunigen.

\section{》) Das Nebenwir-} kungsmanagement bei Immuntherapie soll in die erweiterte S3-Leitlinie Supportivtherapie aufgenommen werden

best practice onkologie: Sie waren maßgeblich daran beteiligt, dass es heute überhaupt S3-Leitlinien zur Supportivtherapie gibt: Kommen die Empfehlungen in der täglichen Praxis flächendeckend an?
K. Jordan: Die Umsetzung der Leitlinien in der breiten Fläche bleibt die große Herausforderung. Bereits 2012 - also vor der S3-Leitlinie Supportive Therapie - zeigte eine Untersuchung, dass nur $29 \%$ der Patienten korrekt nach antiemetischen Leitlinien behandelt wurden [5]. Die Annahme, dass es über die Jahre besser geworden sei, ist leider falsch. Auf der Jahrestagung der ASCO 2019 wurde eine Arbeit präsentiert, die die Anpassung von nationalen Leitlinien der ASCO und NCCN zur prophylaktischen Antiemese von Carboplatin in nur etwa $20 \%$ der Fälle abbildet [6]. Ich glaube, dass die flächendeckende Implementierung der Leitlinien eine der maßgeblichen Aufgaben ist, vor der wir in der Supportivtherapie stehen.

best practice onkologie: Wie kann es gelingen, die supportive Therapie routinemäßig in die Behandlung zu implementieren? K. Jordan: Die erste Idee ist immer, Fortbildungsmaßnahmen zu fördern - das kann ein Baustein der Implementierung sein, reicht allein aber leider nicht aus. Sinnvoll wäre es, den viel diskutierten Prozess der Digitalisierung zu nutzen: Die erforderliche klassische Supportivtherapie sollte direkt im (Chemo-)Therapieprotokoll hinterlegt sein. Automatismen könnten hier den Informationsverlust verhindern. Elegant wäre es, wenn sich jetzt nicht jede einzelne Praxis oder Klinik individuell Protokolle inklusive Supportivmedikation erarbeiten muss, sondern auf ein einheitliches Protokollsystem zurückgreifen kann. Das wäre für den Einzelnen hilfreich und in der Gesamtheit sicher ein qualitativer Zugewinn. Bestrebungen dazu gibt es bereits, eine flächendeckende Umsetzung ist aber noch weit entfernt.

\section{》) Die flächendeckende Implementierung der Leitlinien ist eine der maß- geblichen Aufgaben der Supportivtherapie}

best practice onkologie: Müssen die Leitlinien auch praktikabler aufbereitet werden?

K. Jordan: Ja, auch das ist ein relevanter Aspekt. Die S3-Leitlinie Supportivtherapie umfasst ohne den Leitlinienreport und die Evidenztabellen bereits 558 Seiten. Dies ist im Grunde unpraktikabel, und ich fürchte, dass niemand sich dieser umfänglichen Lektüre in Gänze annehmen wird. Wir brauchen aber beides - kurze, schnell zu erfassende Informationen und Algorithmen für die tägliche Nutzung und - das ist das Wertvolle der S3-Leitlinien - die diskutierte Zusammenfassung der Evidenz, wie sie in ihrer Ausführlichkeit in den S3-Leitlinien nachzulesen ist. Dann können die Behandler diffizilere Entscheidungen treffen.

\section{》) Wichtig ist, dass die klassische prophylaktische Supportivtherapie direkt in das Chemotherapiepro- tokoll eingetragen ist}

best practice onkologie: Wie stellen Sie in Ihrer Klinik sicher, dass neue Erkenntnisse der Supportivtherapie umgesetzt werden?

K. Jordan: Wir haben zunächst eine Arbeitsgruppe Supportivtherapie an meinem neuen Standort am Universitätsklinikum Heidelberg gebildet. Gerade haben wir die aktuellsten Empfehlungen der 
Supportivtherapie systematisch in die einzelnen Chemotherapieprotokolle implementiert und adressieren somit die wichtigsten zu erwartenden Nebenwirkungen. Es muss aber auch Neues hinzukommen. Wir sind jetzt dabei, die Symptome der Patienten systematisch und elektronikbasiert nach dem Prinzip des „patientreported outcome“ abzufragen und standardisiert zu erfassen. Dies ermöglicht zweifelsohne höhere Vigilanz für therapieassoziierte Symptome und eine raschere Behandlung der Nebenwirkungen. Hierbei ist mir die Arbeit im interdisziplinären Team auch mit der Klinikapotheke und unseren Informatikern sehr wichtig. Eine systematische elektronische Implementierung, die auch eine entsprechende Symptommanagementstrategie impliziert und dazu noch praktikabel ist, ist wirklich alles andere als trivial.

best practice onkologie: Für das $\mathrm{Ma}$ nagement der Chemotherapie-induzierten Polyneuropathien (CIPN) empfehlen Sie ein regelmäßiges Funktionstraining [7]. Wie funktioniert das?

K. Jordan: Trotz zahlreicher und qualitativ guter Studien gibt es bislang keine effektive medikamentöse Prophylaxe der CIPN. Dafür gibt es klare Hinweise auf eine Wirksamkeit von nichtmedikamentösen Methoden zur Reduktion einer Polyneuropathie (PNP). Dass bewegungstherapeutische Interventionen polyneuropathische Beschwerden reduzieren können, ist seit längerem für die diabetische Poly-

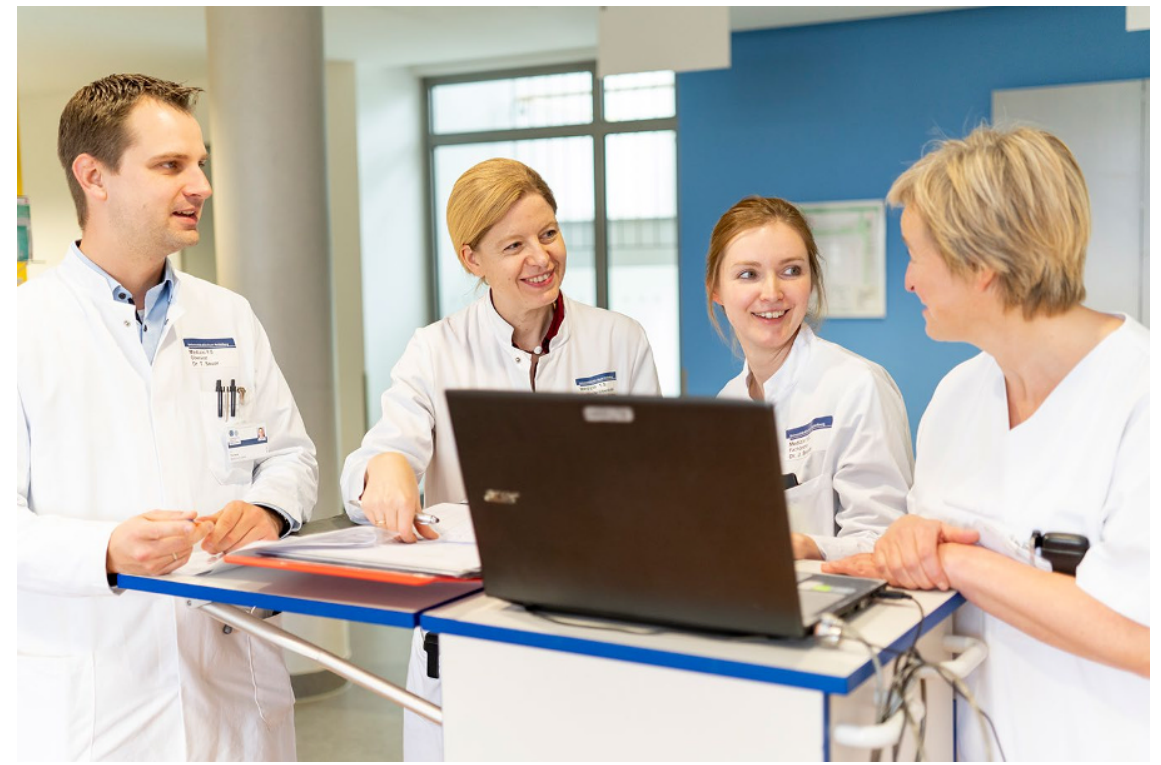

In der täglichen Visite gilt es, schon früh Hinweise auf mögliche unerwünschte Wirkungen unter der onkologischen Behandlung wahrzunehmen und anzusprechen

neuropathie untersucht, für onkologische Patienten mit therapieinduzierter Neuropathie ist die Datenlage jedoch noch dünner. Aber ein 2-mal wöchentlich durchgeführtes Trainingsprogramm, bestehend aus Kraft-, Ausdauer- und koordinativen/ sensomotorischen Übungen, kann nachweislich nicht nur koordinative Fähigkeiten verbessern, sondern beeinflusst auch neurologische Parameter wie Vibrationsempfinden und Tiefensensibilität. Dass es sinnvoll ist, nicht abzuwarten, bis die Symptomatik entsteht, sondern bereits während der onkologischen Therapie zu

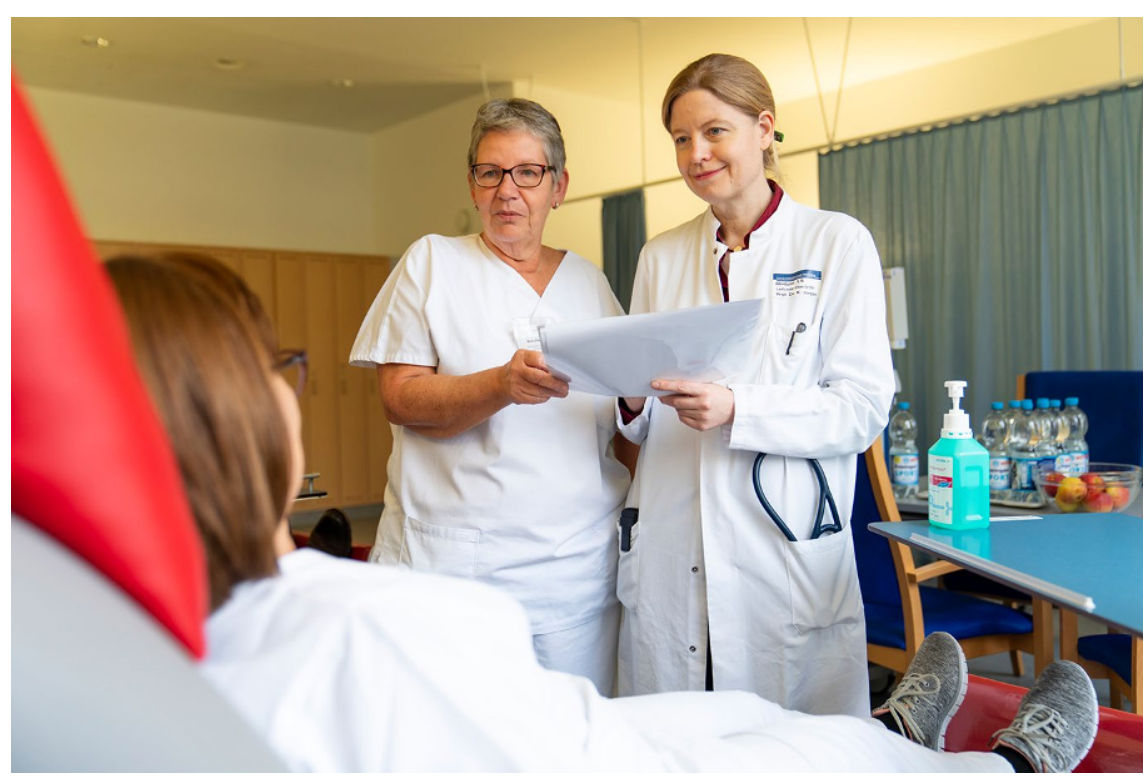

Dem Patienten gilt die volle Aufmerksamkeit trainieren, erscheint logisch, ist aber noch nicht durch größere Studien belegt. Aktuell sind Balancetraining, Gleichgewichtsübungen, Übungen für die Feinmotorik, aber auch Pilates oder Tai-Chi empfohlen. Die vielfältigen positiven Auswirkungen der onkologischen Sporttherapie werden gerade in zahlreichen Studien untersucht, beispielhaft seien die Forscherteams um PD Dr. Sportwiss. Freerk Baumann in Köln und um PD Dr. Joachim Wiskemann in Heidelberg genannt. Ich hoffe, dass die entstehende Evidenz in naher Zukunft in einer S3-Leitlinie Bewegungstherapie aufbereitet wird.

best practice onkologie: Gibt es neben der Bewegungstherapie noch andere Möglichkeiten, um der CIPN vorzubeugen?

K. Jordan: Im Moment laufen kleinere Studien zur Kryo- und zur Kompressionstherapie. Bei der Kryotherapie werden die Hände während der Infusion der chemotherapeutischen Medikamente gekühlt. Für die Kompressionstherapie zieht der Patient während der Behandlung etwas zu kleine chirurgische Handschuhe an. Durch die Eindämmung des Blutflusses in die Hände tritt die CIPN möglicherweise seltener auf.

best practice onkologie: Lassen sich noch weitere Nebenwirkungen mit Hilfe der Bewegungstherapie lindern?

K. Jordan: In Studien belegt ist vor allen Dingen die Auswirkung auf Fatigue. Das 
ist eine sehr belastende Nebenwirkung, die mitunter bei $80 \%$ der Patienten unter der antineoplastischen Therapie auftritt. Hier ist die Bewegungstherapie allen anderen, auch den medikamentösen, Interventionen überlegen.

\section{I) Man darf Apps in der Supportivtherapie nicht überschätzen}

best practice onkologie: Was halten Sie von digitalen Hilfsmitteln für Patienten wie Apps, die zum Sport animieren. Könnten diese die Supportivtherapie bereichern?

K. Jordan: Ja, ich bin mir sicher, dass solche Apps hilfreich sein können. Man darf die „neue Technik“ im Allgemeinen aber auch nicht überschätzen. Nehmen wir z. B. die Compliance. In der Studie SWOG S1105 [8] wurden Brustkrebspatientinnen 2-mal pro Woche per SMS daran erinnert, ihre Aromataseinhibitoren einzunehmen. Genützt hat es leider nichts, die Patientinnen nahmen daraufhin ihre Medikamente nicht stringenter ein, die Compliance verbesserte sich somit nicht. Digitale Hilfsmittel muss man also grundsätzlich sehr kritisch prüfen. Ein besserer Effekt ließe sich vielleicht ableiten, wenn die Hinweise individualisiert auf die Erkrankung zugeschnitten wären. Und wenn letztlich die App wieder eine Verknüpfung zur Versorgung in der sogenannten "realen Welt" herstellt, also z. B. die in der App angegebenen Symptome beim nächsten Ambulanztermin besprochen werden oder bei schweren angegebenen Problemen ein telefonischer Kontakt zum Behandler erfolgt. Damit wären sie auch speziell im Supportivtherapiebereich sicher sehr gut einsetzbar.

best practice onkologie: Frau Prof. Jordan, vielen Dank für das interessante Gespräch.

Das Interview führte: Sabrina Kempe, Dresden

Fotos: Carsten Büll
Literatur

1. Leitlinienprogramm Onkologie (2017) S3-Leitlinie „Supportive Therapie bei onkologischen Patient/nnen"

2. Jordan Ket al (2018) European Society for Medical Oncology (ESMO) position paper on supportive and palliative care. Ann Oncol 29(1):36-43

3. Jordan Ket al (2015) Recent developments in the prevention of chemotherapy-induced nausea and vomiting (CINV): a comprehensive review. Ann Oncol 26:1081-1090

4. Darmani N et al (2019) A new target for future antiemetics: inhibition of glycogen synthase kinase 3 (GSK3). Support Care Cancer 27(1):S30

5. Aapro M et al (2012) The effect of guideline-consistent antiemetic therapy on chemotherapy-induced nausea and vomiting (CINV): the Pan European Emesis Registry (PEER). Ann Oncol 23(8):19861992

6. Navari RM et al (2019) Physician concordance with update to ASCO guidelines for antiemetic use with carboplatin AUC $\geq 4$. J Clin Oncol 37(suppl 15):11595

7. Jordan Ket al (2017) Supportive treatments for patients with cancer. Dtsch Arztebl Int 114(2728):481-487

8. Hershman DL et al (2019) Randomized trial of text messaging (TM) to reduce early discontinuation of aromatase inhibitor (AI) therapy in women with breast cancer: SWOG S1105. I Clin Oncol 37(suppl 15):6516

\section{Lesetipp}

\section{Präzisionsmedizin beim Lungenkarzinom}

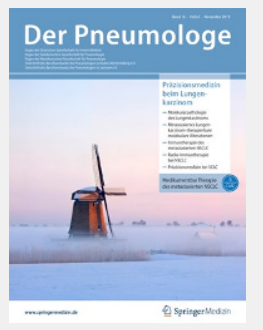

Neben der klassischen Pathologie hat sich mit der Molekularpathologie eine Subspezialität der Pathologie entwickelt, welche die entscheidende Determinante der

pathologischen Untersuchung von Lungentumoren darstellt.

In Der Pneumologe 06/2019 finden Sie aber nicht nur Beispiele, wie komplexe Pathologie auf didaktisch überzeugende Art und Weise einem "normalen Arzt" nähergebracht werden kann. Auch die Wertigkeit der prätherapeutischen Mutationsanalyse wird dargestellt und die Immuntherapie mit Einsatz sog. Checkpoint-Inhibitoren, sowie das Procedere im Falle einer Resistenzentwicklung werden erklärt.

Die Beiträge in diesem Themenheft bieten Ihnen Aktuelles auf der Höhe der Entwicklungen, damit Sie Ihren schwerkranken Patienten die bestmögliche Therapie anbieten können.

- Molekularpathologie des Lungenkarzinoms: aktuelle Standards und weitere Entwicklungen

- Immuntherapie des metastasierten nicht-kleinzelligen Lungenkarzinoms

- Radio-Immuntherapie als neuer Standard?

- Perspektiven der Präzisionsmedizin

Suchen Sie noch mehr zum Thema? Mit e.Med - den maßgeschneiderten Fortbildungsabos von Springer Medizin - haben Sie Zugriff auf alle Inhalte von SpringerMedizin.de. Sie können schnell und komfortabel in den für Sie relevanten Zeitschriften recherchieren und auf alle Inhalte im Volltext zugreifen.

Weitere Infos zu e.Med finden Sie auf springermedizin.de unter „Abos" 